\title{
Modular Transport and Sorting System with Omnidirectional Wheels
}

\author{
Ciprian Ion Rizescu ${ }^{1}$, Andrei Ioan Pleşea ${ }^{1}$, and Dana Rizescu ${ }^{1}$ \\ ${ }^{1}$ University POLITEHNICA of Bucharest, 313 Splaiul Independentei, Bucharest, Romania
}

\begin{abstract}
The paper refers to the development of an intelligent sorting and transport system. This research aims to develop such systems and solve problems in multi-directional transport as well as the development of a sorting, transport and positioning system that can be used in various fields. At the same time, the omnidirectional transport system is a modular transport and sorting system. It consists of several small hexagonal transport cells, each with three individually operated omnidirectional wheels. By individually controlling the wheels, objects can be transported freely in all directions. This opens unlimited possibilities to create new concepts for handling materials or to improve existing classic ones. Due to the modular construction and the individual control of the omnidirectional wheels, the sorting tasks can be adapted very quickly. This will allow you to perform different tasks in very small areas. Individual control of objects also allows for exact alignment or individual rejection of objects. The system has been designed to transport work objects. To design the module, it was necessary to create its scale geometric model using the Autodesk Inventor Professional 2018 design environment. To make the module, it was used through the rapid prototyping process with Fused Deposition Modelling, the material used being Polylactic Acid. The solution adopted allows any transported object to always be supported on at least three wheels, this allows the direction of movement of the object to be controlled by the movement of the wheels.
\end{abstract}

\section{Introduction}

This research aims to develop modular transport systems and solve problems in multidirectional transport as well as the development of a sorting, transport and positioning system that can be used in various fields. A system has been designed to transport work objects. The system consists of several hexagonal cells each with three individually controlled omnidirectional wheels. To design the module, it was necessary to create its scale geometric model using the Autodesk Inventor Professional 2018 design environment. To make the module, it was used through the rapid prototyping process with FDM (Fused Deposition Modelling), the material used being PLA (Polylactic Acid).

Through its simple structure, the system can be flexibly adapted to the most diverse requirements and offers endless possibilities. It is also very easy and cheap to maintain and with its intelligent control, it will become a basic element for the digital factory of tomorrow. 
Flexible automation technology plays a more important role in goods logistics. The innovative features of a space-saving conveyor system, with diverse and flexible application options, can be used in the growing order market or in the intralogistics market $[1]$.

The solution adopted allows any transported object to always be supported on at least three wheels, this allows the direction of movement of the object to be controlled by the movement of the wheels. Conveyor systems not only carry materials, but also perform various sorting tasks, such as cantoning, positioning, or rejecting / sorting. Conveyor roller systems require separate sections for various tasks. Executing the transport on one direction or change of direction of travel involves a long-distance system with large areas and complex tasks. Therefore, transport systems are proving less flexible for modernization and the execution of more complex tasks. Moreover, this omnidirectional transport system allows objects to move freely and independently of each other in the plane. Due to the modular construction and the individual control of the omnidirectional wheels, the sorting tasks can be adapted very quickly. This will allow you to perform different tasks in very small areas. Individual control of objects also allows for exact alignment or individual rejection of objects $[2,3]$.

\section{Modular transport system}

The authors designed a system to transport work objects. The system consists of several hexagonal cells each with three individually controlled omnidirectional wheels. In Fig. 1 shows the block diagram of the modular transport system. Using a common PC, it is possible to control the system through a special module designed for the user. This allows you to set the desired path for work objects by entering the drive parameters. The information entered the PC is then sent to the control unit (PLC). The control unit sends the control signals to the motors so that the objects can be transported on the desired route. This system can be used in parallel in sorting applications [4-6].

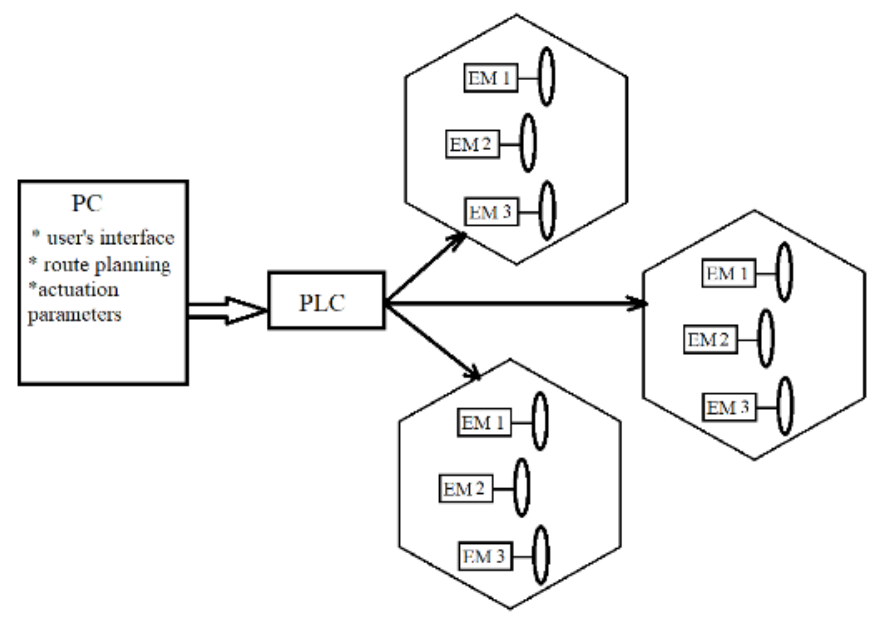

Fig. 1. Block sketch of the modular transport system.

Each conveyor module has in its component an electric motor, a reducer (gear unit), and a drive wheel for the workpieces. Fig. 2 shows the functional diagram of the entire module system. In this paper is considered the operation of 9 hexagonal modules with a total of 27 stepper motors controlled by Arduino Nano development boards. These 9 Arduino Nano boards will be controlled by an Arduino Uno development board having the role of Master 
in $\mathrm{I} 2 \mathrm{C}$ communication. The 9 modules were considered sufficient to study the transport system and the sorting of parts system. In Fig. 3 is presented the sketch of the reducer and a view of the reducer, which consists in 7 gears. The reducer ratio is 16 .

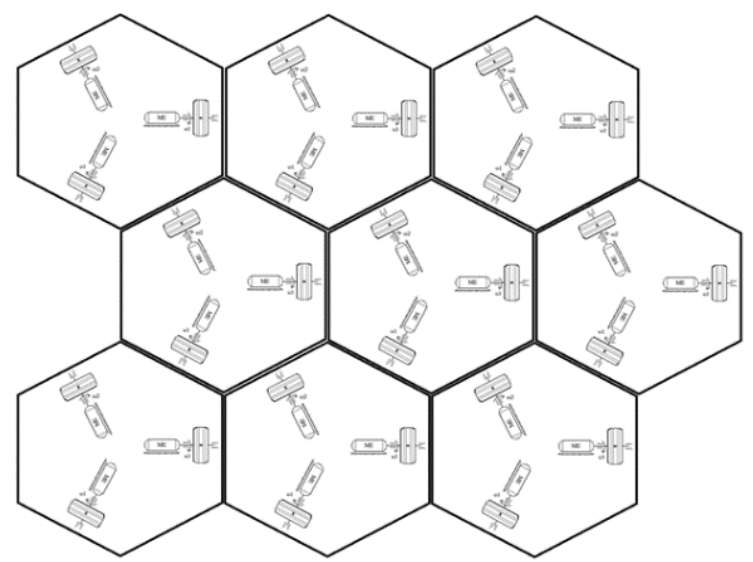

Fig. 2. Functional diagram of the 9 modules.
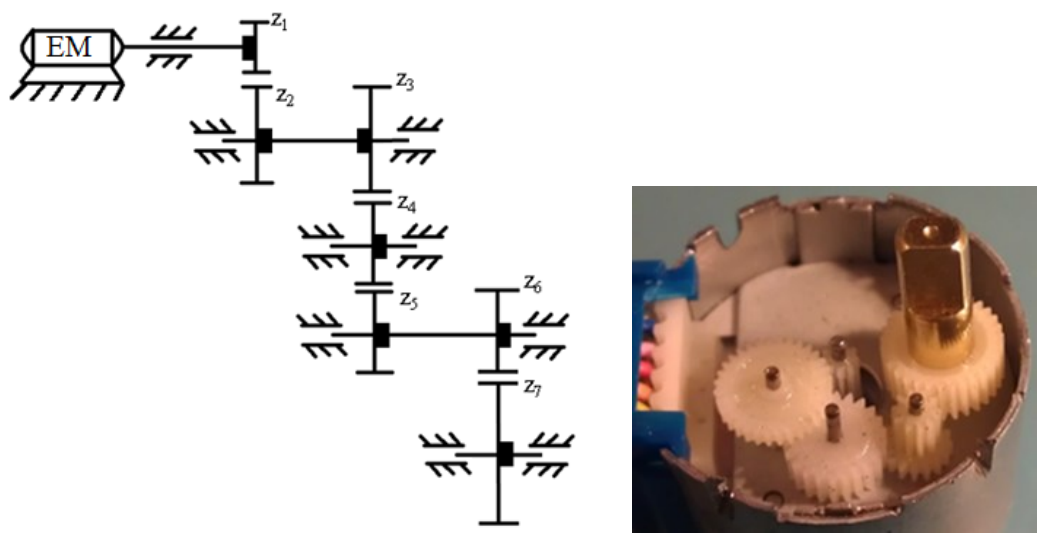

Fig. 3. Cinematic sketch of the reducer and the reducer assembly.

The 28BYJ-48 stepper motor was chosen for driving the wheels. The easiest way to control a unipolar stepper motor is with the help of the Arduino development board, a ULN2003 driver is also required to make the connection between the motor and the Arduino. This driver amplifies the Arduino signal and consists of seven pairs of Darlington transistors as well as internal diodes to dissipate the voltage when driving inductive loads.

To develop the module, it was necessary to create the geometric model at its scale using the Autodesk Inventor Professional 2018 design environment. Fig. 4 shows the designed geometric model. After transmitting the information to the machine tool, the part was made in about 10 hours. Fig. 5 shows the printing result using rapid prototyping technology $[7,8,13]$. Ender 3 - 3D printer with $1.75 \mathrm{~mm}$ Polaroid Premium filament was used to make this module.

The Inter Integrated Circuit (I2C) protocol is a protocol created to allow multiple "slave" integrated circuits to communicate with one or more "master" chips. This type of communication can only be used over short communication distances and like the UART protocol it only needs 2 signal wires to send / receive information. 


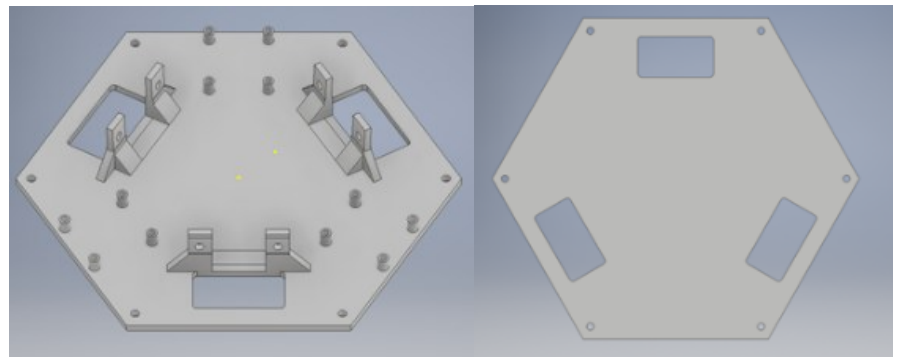

Fig. 4. Design the module using AutoCAD 2018.

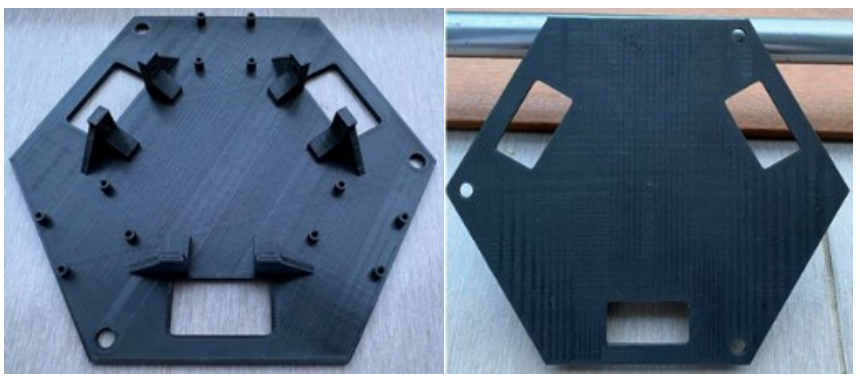

Fig. 5. 3D Printed module with 3D Ender 3 printer.

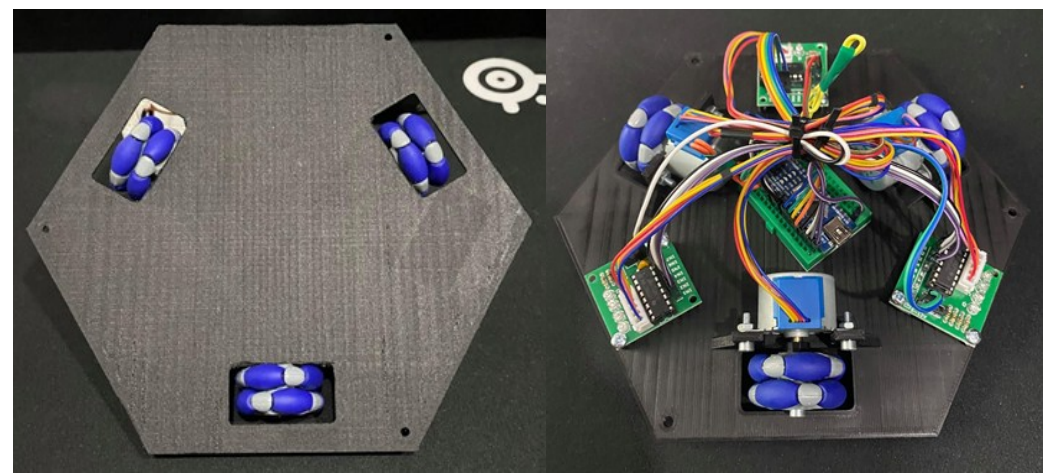

Fig. 6. One module full equipped

To simulate the operation of a module, the authors started from a single stepper motor, reducer, driver, for which a principal scheme was elaborated, which was then simplified. The result is presented in Fig. 7. Each module consists of three stepper motors, three reducers, three drivers and an Arduino. By implementing the equivalent scheme for a stepper motor in Fig. 7 in the Simulink environment, the operation simulation scheme in Fig. 8 is obtained.

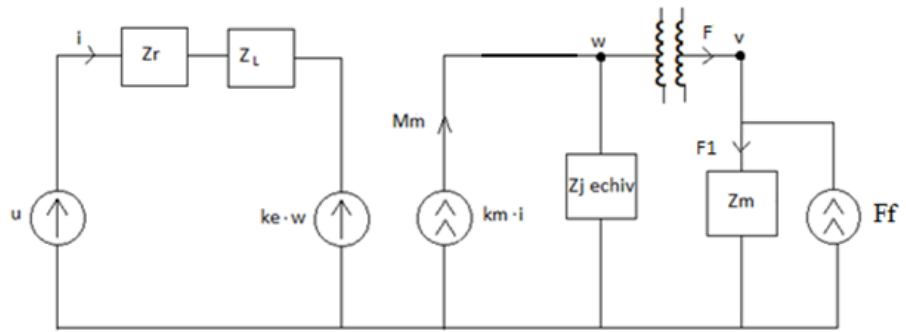

Fig. 7. Simplified equivalent scheme. 


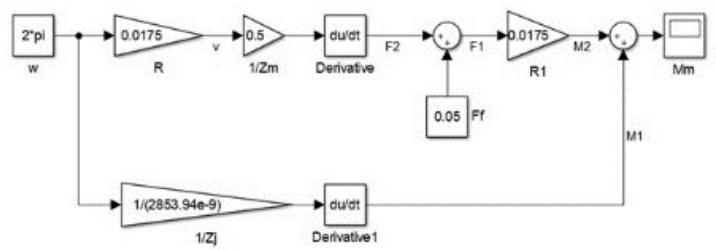

Fig. 8. Simulation of operation for a stepper motor.

With the help of the open-source program Fritzing, the electrical diagram was made in which the connection of the components is highlighted as shown in Fig. 9.

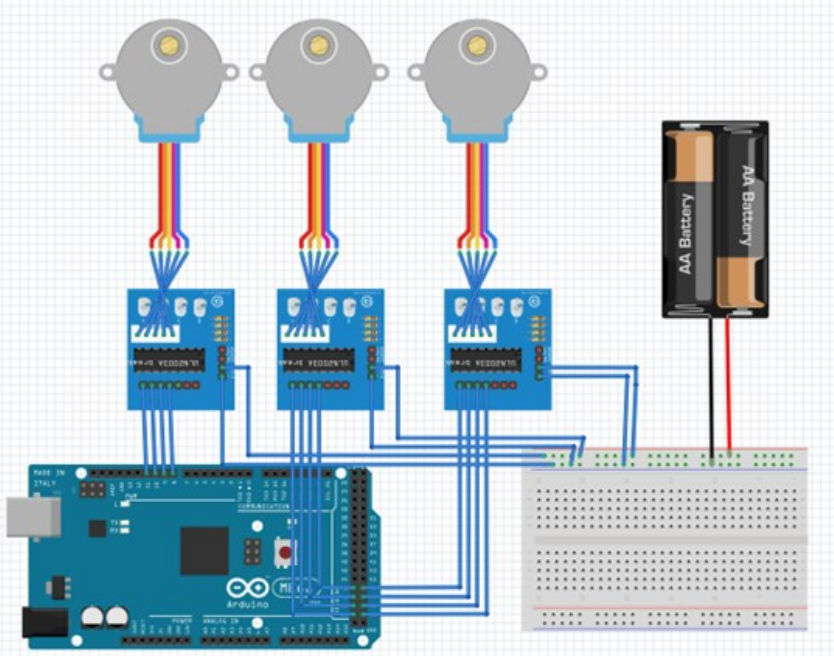

Fig. 9. Wiring diagram for the three stepper motors mounted on a module.

A connection bridge was built to connect all modules to the I2C network. Figure 10 shows the connections for 3 modules. To control all modules (9) a Master program installed via the Arduino platform on an Arduino Uno was required.

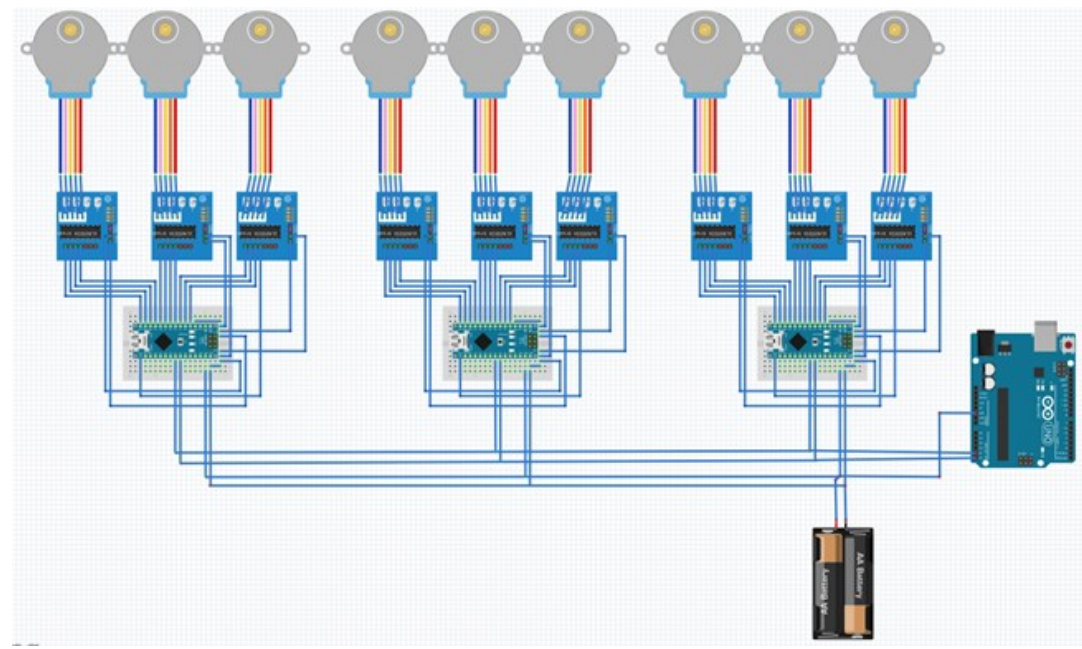

Fig. 10. Wiring diagram for three modules 


\section{Transport selection mode}

As can be seen in Fig. 11 - left, all three wheels move in the same direction, which causes the object to rotate. In a situation where the object entered on the conveyor belt must have a different orientation at the exit from it, this example shows how the orientation of an object can be changed [9-11]. The arrangement of the wheels was considered with $120^{\circ}$ between the axles of the wheels. The wheels outer diameter is $52 \mathrm{~mm}$.

Fig. 11 - centre presents the situation when the objects must be transported in the forward direction. This is possible by controlling two wheels side by side with rotational movements opposite each other, which causes the object to move in the forward direction as the blue arrow is oriented. This shows the second type of movement of objects in which the objects move forward.

In the third wheel control mode, shown in Fig. 11 - right, the direction of movement of the object, expressed with the blue arrow, will follow the direction of movement of the wheel placed on the left side. It is necessary to control the other two wheels with controls opposite to the first and at a lower speed. This wheel control is very useful for the sorting process.

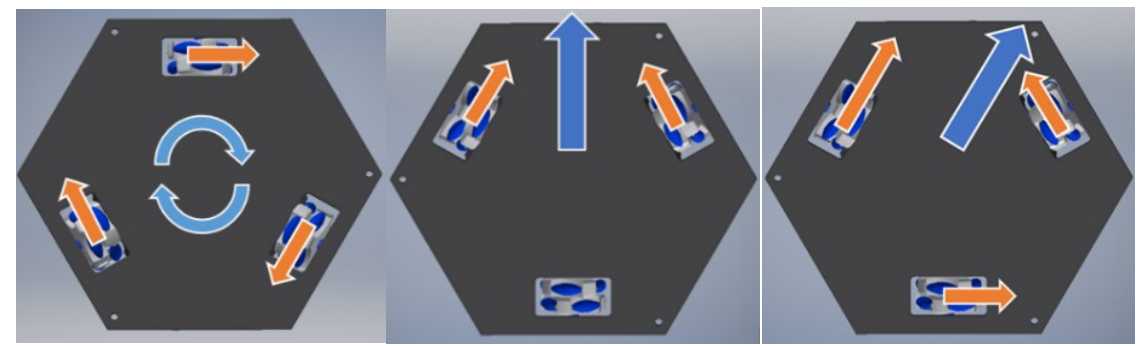

Fig. 11. Rotation, forward and turning to right transport directions.

The last type of displacement, namely selective displacement. This type is characterized by changing the trajectory of the transported product to the desired area, by operating the motors as it was presented in all three Fig. 11 pictures.

\section{Experimental setup}

During the experimental setup tests, a route of a box was made on the module table, Fig. 12. This route has been predetermined and follows the trajectory of a lateral edge of the table, after which in the end it rotates and turns on the other lateral edge of the table of the modules. This route is presented in Fig. 13 both left and right. The workpiece (box) weight is about $2 \mathrm{~kg}$. For experimental setup, a box with sizes of 100x150x100 (WxLxH) mm was chosen as a reference. Since three collinear points form a plane, and this system aims to move objects on the plane of the wheels, it is necessary that the distances between the wheels are chosen accordingly. The dimensions of the outer plate have been chosen in relation to the reference box, more precisely, the box must always be supported on any three wheels of the conveyor system. Several variants were also considered for the route of the parts, but the most difficult are those with sudden route changes [12]. 


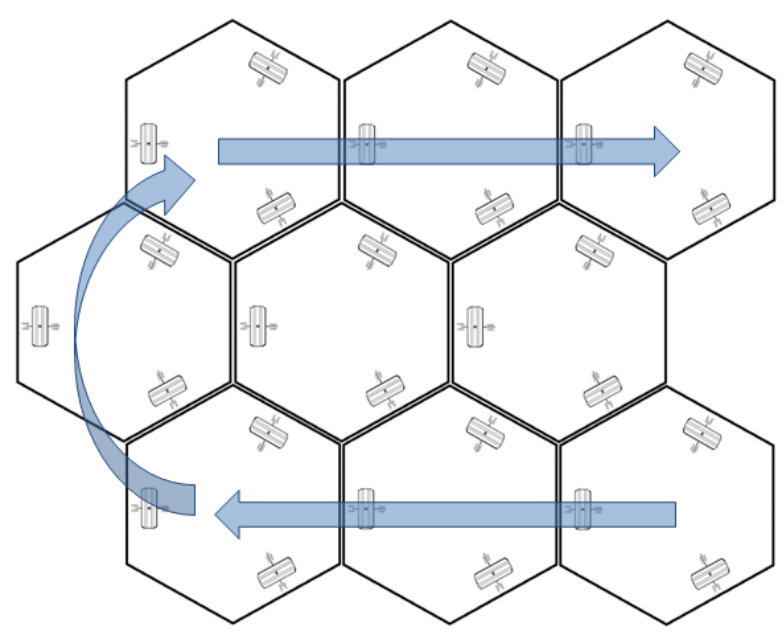

Fig. 12. Example of a route tested on experimental setup.

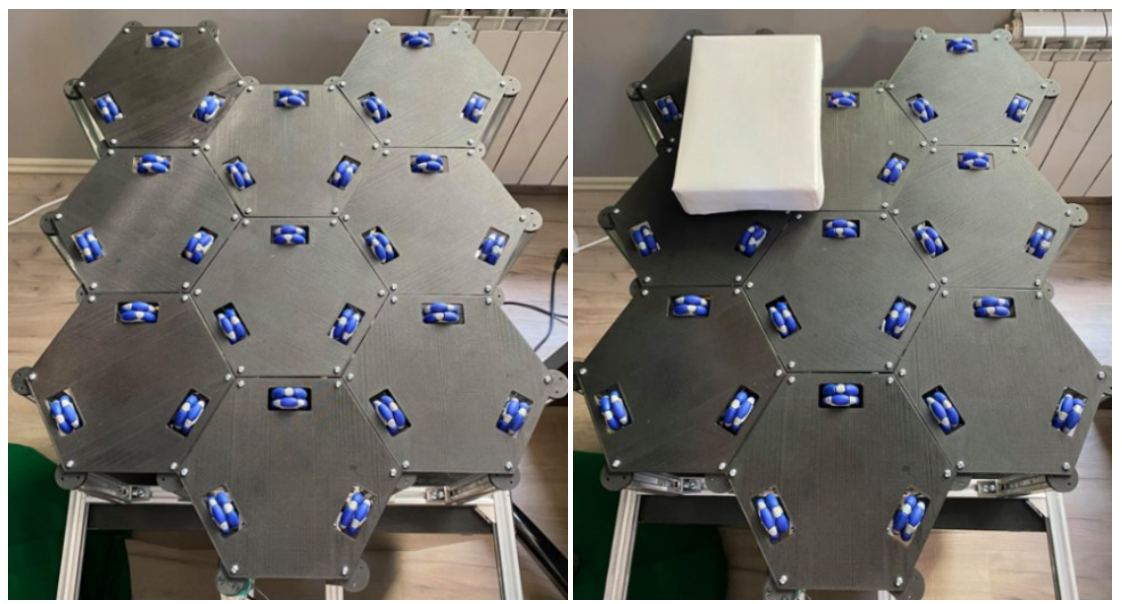

Fig. 13. Rotation, forward and turning to right transport directions.

\section{Conclusions}

The main advantage of a modular transport and sorting system with omnidirectional wheels is the significant reduction in the size of a conventional conveyor belt.

Another important advantage of such a system is the flexibility because modules can be added or removed depending on the user's requirements. It can thus be stated that the set of modules formed does not have a fixed size, therefore it can be placed in various work areas. In the event of a malfunction in a multi-module conveyor belt, the replacement of the malfunctioning module is quick and easy. At the same time, this system allows the simultaneous manipulation of several objects with the help of omnidirectional modules and wheels arranged uniformly on the entire surface of the work belt. Following the practical realization and testing, the need for sensors to identify the position of the box at each moment was identified. Also, making guides around the table would have helped in the direction of palletizing the boxes. In conclusion, the ability to move objects simultaneously and independently of each other, the modular structure, the excellent adaptability of the 
system to new tasks, as well as the use of small spaces meet the current requirements of many companies.

\section{References}

1. https://cjme.springeropen.com/articles/10.1007/s10033-017-0164-7, (Accessed 15.05.2021)

2. http://www.intralox.com/arb-automation-equipment.aspx, (Accessed 15.05.2021)

3. http://www.intralox.com/automotive-manufacturing.aspx, (Accessed 15.05.2021)

4. https://www.multi-conveyor.com/packaging-solutions/activated-roller-belt-conveyors, (Accessed 15.05.2021)

5. http://www.mhi.org/media/members/14506/130020635014972950.pdf, (Accessed 15.05.2021)

6. https://www.interroll.com/blog/looking-beyond-our-horizons-interrolls-productionsystem/, (Accessed 15.05.2021)

7. https://www.rotacaster.com.au/shop-product/35mm-rotacaster-wheels/rotacaster35mm-double--35a--tpe-roller--abs-body-and-bushing, (Accessed 15.05.2021)

8. http://www.isim.ro/tima/past_tima/docs/past_tima/papers_tima09.htm, (Accessed 15.05.2021)

9. https://www.interroll.ca/en/products/drives-and-controls/rollerdrive/rollerdrive-ec100/, (Accessed 15.05.2021)

10. https://patents.google.com/patent/DE102012014181A1/en, (Accessed 15.05.2021)

11. D. Cordoneanu, C.Nitu, ICOMECYME Networks Systems 48 (2018)

12. J.B. Song, K.S. Byun, Design and Control of an Omnidirectional Mobile Robot with Steerable Omnidirectional Wheels, www.i-techonline.com, (Accessed 15.05.2021)

13. D. Rizescu, C.I. Rizescu, ICOMECYME Networks Systems 20, 48 (2018) 